Article

\title{
Theoretical Calculation of the Gas-Sensing Properties of Pt-Decorated Carbon Nanotubes
}

\author{
Xiaoxing Zhang ${ }^{1{ }^{*},}$, Ziqiang Dai ${ }^{1}$, Li Wei ${ }^{2}$, Naifeng Liang ${ }^{2}$ and Xiaoqing Wu ${ }^{1}$ \\ 1 State Key Laboratory of Power Transmission Equipment \& System Security and New \\ Technology, Chongqing University, Shapingba District, Chongqing 400044, China; \\ E-Mails: daizq382812@163.com (Z.D.); wufeng.200809@163.com (X.W.) \\ 2 State Grid Xinjiang Electric Power Company, Construction Road, Urumchi 830011, China; \\ E-Mails: liweicqu2007@hotmail.com (L.W.); Liangnaifeng@xj.sgcc.com.cn (N.L.) \\ * Author to whom correspondence should be addressed; E-Mail: zhxx@cqu.edu.cn; \\ Tel.: +86-136-4055-1418; Fax: +86-023-6510-2442.
}

Received: 11 August 2013; in revised form: 10 October 2013 / Accepted: 24 October 2013 / Published: 6 November 2013

\begin{abstract}
The gas-sensing properties of Pt-decorated carbon nanotubes (CNTs), which provide a foundation for the fabrication of sensors, have been evaluated. In this study, we calculated the gas adsorption of Pt-decorated $(8,0)$ single-wall CNTs (Pt-SWCNTs) with $\mathrm{SO}_{2}, \mathrm{H}_{2} \mathrm{~S}$, and $\mathrm{CO}$ using GGA/PW91 method based on density functional theory. The adsorption energies and the changes in geometric and electronic structures after absorption were comprehensively analyzed to estimate the responses of Pt-SWCNTs. Results indicated that Pt-SWCNTs can respond to the three gases. The electrical characteristics of Pt-SWCNTs show different changes after adsorption. Pt-SWCNTs donate electrons and increase the number of hole carriers after adsorbing $\mathrm{SO}_{2}$, thereby enhancing its conductivity. When $\mathrm{H}_{2} \mathrm{~S}$ is adsorbed on CNTs, electrons are transferred from $\mathrm{H}_{2} \mathrm{~S}$ to Pt-SWCNTs, converting Pt-SWCNTs from $p$-type to $n$-type sensors with improved conductivity. However, Pt-SWCNTs obtain electrons and show decreased conductivity when reacted with $\mathrm{CO}$ gas.
\end{abstract}

Keywords: platinum-decorated carbon nanotubes; density functional theory; frontier orbital 


\section{Introduction}

Carbon nanotubes (CNTs) have structures with abundant pores, large surface-to-volume ratios, and strong adsorption and desorption capabilities for gases. Gas molecules that adsorb on the surface of CNTs change the shape of CNTs and trigger redistribution of electrons, leading to a macroscopic change in resistance [1]. Kong et al. [2] used chemical vapor deposition (CVD) to fabricate single-wall CNTs (SWCNTs) on $\mathrm{SiO}_{2} / \mathrm{Si}$ substrates to detect $\mathrm{NO}_{2}$ (2 ppm to $200 \mathrm{ppm}$ ) and $\mathrm{NH}_{3}$ $(0.1 \%$ to $1 \%)$ diluted in air or Ar. Results showed that the conductivity of SWCNTs decreases threefold after adsorbing $\mathrm{NH}_{3}$, whereas the conductivity increases threefold after adsorbing $\mathrm{NO}_{2}$. Unlike traditional gas sensors, CNT gas sensors exhibit faster response, higher sensitivity, smaller size, and lower working temperatures [3,4]. These advantages make CNTs suitable for application in industries, the medical field, and environmental protection. Recently, the CNT gas sensor has received extensive research attention and achieved certain interesting results. It is significant to study the gas sensitive properties of CNTs, which is the foundation of sensor design. In this paper, the gas sensitive property of CNTs is studied mainly through theoretical calculation analysis. Intrinsic CNTs can only detect several strong oxidizing and reducing gases, while other gases are only weakly adsorbed with low sensitivity because of the structure and chemical properties of intrinsic CNTs [5-7]. To overcome this limitation, some researchers have proposed various physical and chemical modifications, such as introduction of new active sites on the surface of CNTs. The authors in [8] showed that polar groups $\left(\mathrm{COOH}, \mathrm{NH}_{2}, \mathrm{NO}_{2}\right.$ and $\left.\mathrm{H}_{2} \mathrm{PO}_{3}\right)$ are promising candidates for enhancing $\mathrm{CO}_{2}$ and $\mathrm{CH}_{4}$ adsorption capacity by strengthening adsorption and activating exposed edges and terraces to introduce additional binding sites. Peng et al. [9] found B-doped CNT gas sensors have a good sensitivity to $\mathrm{CO}$ and $\mathrm{H}_{2} \mathrm{O}$. Besides, transition metals are rich in $d$-electrons and empty orbitals, wherein small gas molecules can bond strongly to the metal when adsorbed on the surface [10,11]. Studies [12] indicated that compared with intrinsic CNTs, CNTs with metal depositions have better sensitivity. For instance, Pt- and Au-functionalized CNTs are more sensitive by an order of magnitude for $\mathrm{NO}_{2}$ and $\mathrm{NH}_{3}$ detection than intrinsic CNTs. The response characteristics of sensors largely depend on the number of active sites, which can strengthen the response of sensors [11]. Pt can adsorb small molecules [13-16], and Pt has a good catalytic activity. CNTs have excellent physical and chemical properties and unique structures that can be used as a supporting material that influences the activity of Pt catalysts [17]. Conversely, the catalytic activity of Pt can improve the gas sensing properties of CNTs. Therefore the present study introduces $\mathrm{Pt}$ as a new active site. The adsorptive processes and properties of Pt-SWCNTs for $\mathrm{SO}_{2}, \mathrm{H}_{2} \mathrm{~S}$, and $\mathrm{CO}$ are calculated. These gases are highly toxic to the human body, making this research significant by providing theoretical support and a good foundation for the fabrication of suitable CNT-based sensors.

\section{Computation Model and Methods}

We built $(8,0)$ SWCNTs and gas molecule models using Materials Studio (Accelrys, San Diego, CA, USA), a molecular dynamics simulation software. The geometries and properties of the system were derived using the quantum mechanics program $\mathrm{DMol}^{3}$ code (Accelrys). We adopted GGA to treat the electronic exchange and correlation effects, as described by PW91 [18]. Pt is a heavy metal 
with an atomic number of 78; therefore, DFT semi-core pseudopotentials [19] were used to manage the interactions between the nucleus and the valence electron. To ensure accuracy, the energy threshold and self-consistent field convergence criteria were set to $2.72 \times 10^{-4}$ and $2.72 \times 10^{-5} \mathrm{eV}$, respectively. The space orbital cutoff radius was set to $0.40 \mathrm{~nm}$, whereas the Brillouin zone k-point sampling was performed in a $1 \times 1 \times 2$ [20,21] Monkhorst-Pack mesh. A $2.50 \mathrm{~nm} \times 2.50 \mathrm{~nm} \times 0.85 \mathrm{~nm}$ periodic boundary was adopted to avoid the interaction between adjacent cells.

Two SWCNT unit cells were selected as intrinsic CNTs to build Pt-SWCNTs. According to references [11,22], Pt can easily adsorb on the vacancy defects of SWCNTs, and its adsorption energy is $6.400 \mathrm{eV}$, which is greater than that of Pt adsorbed on perfect crystal surface $(2.750 \mathrm{eV})$. Accordingly, the present research selected this Pt-SWCNT model with geometry optimization structure, as shown in Figure 1.

Figure 1. Structural model of Pt-SWCNTs. (a) Front view; (b) side view.

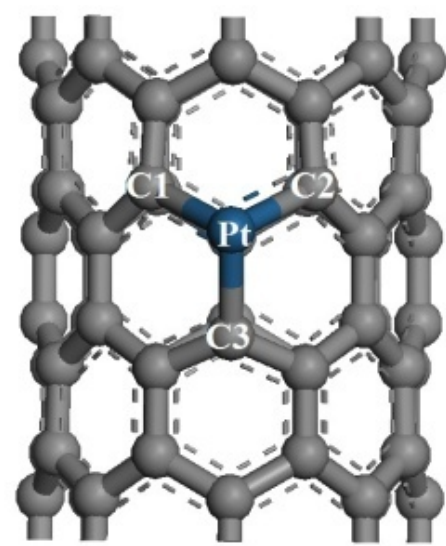

(a)

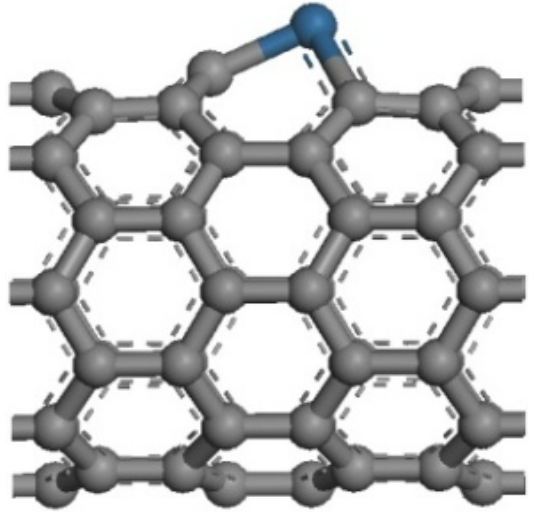

(b)

\section{Results and Discussion}

The radius of a Pt atom is $0.183 \mathrm{~nm}$, which is greater than that of a $\mathrm{C}$ atom $(0.070 \mathrm{~nm})$. Thus, $\mathrm{Pt}$ is highlighted on the CNT surface. The bond lengths between the Pt atom and three adjacent $\mathrm{C}$ atoms changed from $0.142 \mathrm{~nm}$ to $0.199,0.199$, and $0.189 \mathrm{~nm}$ for Pt-C1, Pt-C2, and Pt-C3, respectively. These results are consistent with the reference [22].

The quantum chemical energies of Pt-SWCNTs and gas molecules, as well as the optimized structure of the adsorption systems $\left(E_{P t-S W C N T s}, E_{g a s}\right.$, and $\left.E_{\text {gas-Pt-SWCNTS }}\right)$ were calculated. The adsorption energy $\left(E_{b}\right)$ between a gas molecule and CNTs can be calculated by the following formula:

$$
E_{b}=E_{g a s-P t-S W C N T s}-E_{P t-S W C N T S}-E_{g a s}
$$

At $E_{b}<0$, the energy of the absorption system is less than the total energy of gas molecules and Pt-SWCNTs. Therefore, the reaction is exothermic and spontaneous. Greater adsorption energy releases more energy during the reaction process. However, when $E_{\mathrm{b}}>0$ it is relatively difficult for the reaction to continue because of the energy required. 
In actual practice, the gas-sensitive response of the sensor is evaluated by the changes in electrical characteristics (e.g., resistance) of sensors. Therefore, we also calculated and analyzed the electronic structure of Pt-SWCNTs, gas molecules, and adsorption system. $E_{H O M O}$ and $E_{L U M O}$ represent the highest occupied molecular orbital (HOMO) energy and the lowest unoccupied orbital (LUMO) energy, respectively. $E \mathrm{~g}$ is the difference of $E_{L U M O}$ and $E_{H O M O}$, and $Q$ is the net charge of the system. The parameters are defined as follows:

$$
\begin{aligned}
& E_{L-H}=\left|E_{L U M O}^{(P t-S W C N T s)}-E_{\text {Hомо }}^{(\text {gas })}\right| \\
& E_{H-L}=\left|E_{\text {LUMO }}^{(\text {gas })}-E_{\text {HOMO }}^{\left({ }^{(P t}-S W C N T s\right)}\right|
\end{aligned}
$$

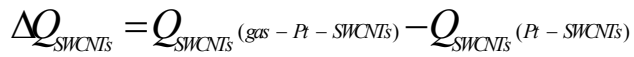

$$
\begin{aligned}
& \Delta Q_{P t}=Q_{P t}(\text { gas }-P t-S W C N T s)-Q_{P t}(P t-S W C N T s)
\end{aligned}
$$

\section{1. $\mathrm{SO}_{2}$}

$\mathrm{SO}_{2}$ is colorless, corrosive, and has a strong pungent odor. Moreover, when $\mathrm{SO}_{2}$ is dissolved in bodies of water, sulfurous acid rain is generated, which is harmful to the environment. $\mathrm{SO}_{2}$ can also form sulfuric acid when dissolved in water, which can irritate the mucous membrane of the eyes and nose.

The full geometric optimization of the Pt-SWCNTs and $\mathrm{SO}_{2}$ adsorption model is shown in Figure 2. An oxygen atom $\mathrm{O} 1$ points to Pt, with Pt-O1 and Pt-S distances of 0.212 and $0.245 \mathrm{~nm}$, respectively. The reaction adsorption energy is $-1.225 \mathrm{eV}$ (Table 1), which denotes an exothermic and spontaneous reaction. By contrast, the reaction adsorption energy of intrinsic SWCNTs is $-0.830 \mathrm{eV}$, so Pt-doping enhances the interaction between $\mathrm{SO}_{2}$ and SWCNTs. Pt is not only a sensing element of Pt-SWCNTs, but also an active site. Strong interaction with gas molecules adsorbed on the surface results in deformation of Pt-SWCNTs and elongation of the Pt-C bond.

Figure 2. Structural model of the $\mathrm{SO}_{2}$-Pt-SWCNT adsorptive system. (a) Front view; (b) side view.

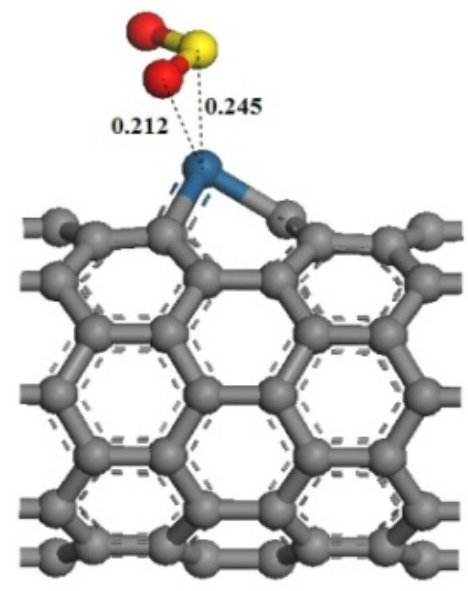

(a)

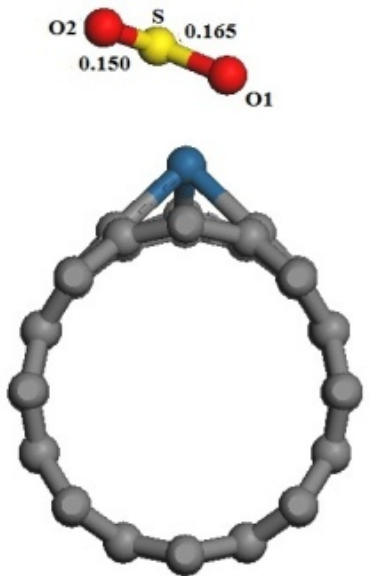

(b) 
Table 1. Adsorption energy and structural parameters of Pt-SWCNTs adsorption.

\begin{tabular}{cccc}
\hline & $\mathbf{S O}_{2}$ & $\mathbf{H}_{\mathbf{2}} \mathbf{S}$ & $\mathbf{C O}$ \\
\hline $\mathrm{d}_{\mathrm{Pt}-\mathrm{C} 1} / \mathrm{nm}$ & 0.205 & 0.200 & 0.205 \\
$\mathrm{~d}_{\mathrm{Pt}-\mathrm{C} 2} / \mathrm{nm}$ & 0.201 & 0.202 & 0.202 \\
$\mathrm{~d}_{\mathrm{Pt}-\mathrm{C} 3} / \mathrm{nm}$ & 0.196 & 0.192 & 0.194 \\
$E_{b} / \mathrm{eV}$ & -1.225 & -0.977 & -1.386 \\
\hline
\end{tabular}

The frontier orbital energy difference of $\mathrm{SO}_{2}$ and Pt-SWCNTs is $E_{H-L} \ll E_{L-H}$. A Pt-SWCNT electron only needs to overcome a $0.158 \mathrm{eV}$ energy barrier to transfer to $\mathrm{SO}_{2}$, whereas a $\mathrm{SO}_{2}$ electron needs to overcome a $3.818 \mathrm{eV}$ energy barrier to transfer to Pt-SWCNTs. Therefore, Pt-SWCNTs provide electrons to $\mathrm{SO}_{2}$ in the adsorption process. A portion of electrons fill the anti-bonding orbital of S-O1, changing the bond length from $0.143 \mathrm{~nm}$ to $0.165 \mathrm{~nm}$. O2 is far from the CNT surface, so the interaction is small, allowing only a small change in the bond length of $\mathrm{S}-\mathrm{O} 2(0.150 \mathrm{~nm})$.

According to the respective Mulliken charge populations, SWCNTs of Pt-SWCNTs have 0.147 positive charge and $\mathrm{Pt}$ has 0.147 negative charge before adsorption. After the adsorption process, SWCNTs have 0.509 positive charge, whereas $\mathrm{Pt}$ has 0.116 negative charge. $\mathrm{SO}_{2}$ obtains 0.393 electrons during the adsorption reaction with Pt-SWCNTs, which is 4.6 times than intrinsic SWCNTs (Table 2). Charge variation $\left(\Delta Q_{S W C N T S}, \Delta Q_{P t}\right)$ of SWCNTs and Pt are 0.362 and 0.031 , respectively (Table 3). Therefore, $\mathrm{SO}_{2}$ obtains electrons mainly from SWCNTs, whereas the Pt exhibits a small charge change.

Table 2. Adsorption energy and charge transform of intrinsic SWCNTs adsorption.

\begin{tabular}{lcc}
\hline & $\boldsymbol{E}_{\boldsymbol{b}} / \mathbf{e V}$ & $\boldsymbol{Q}_{\text {gas }} / \mathbf{e}$ \\
\hline $\mathrm{SO}_{2}$-SWCNTs & -0.830 & -0.067 \\
$\mathrm{H}_{2}$ S-SWCNTs & -0.591 & 0.013 \\
CO-SWCNTs & -0.157 & 0.006 \\
\hline
\end{tabular}

Table 3. Electrical structure parameters of the adsorption structures.

\begin{tabular}{cccccc}
\hline System & $\mathbf{Q}_{\text {SWCNTs }} / \mathbf{e}$ & $\mathbf{Q}_{\mathbf{P t}} / \mathbf{e}$ & $\mathbf{Q}_{\text {gas }} / \mathbf{e}$ & $\Delta \mathbf{Q}_{\text {SWCNTs }} / \mathbf{e}$ & $\Delta \mathbf{Q}_{\mathbf{P}} / \mathbf{e}$ \\
\hline Pt-SWCNTs & 0.147 & -0.147 & & & \\
SO $_{2}$-Pt-SWCNTs & 0.509 & -0.116 & -0.393 & 0.362 & 0.031 \\
$\mathrm{H}_{2}$ S-Pt-SWCNTs & -0.019 & -0.266 & 0.285 & -0.166 & -0.119 \\
CO-Pt-SWCNTs & 0.166 & -0.347 & 0.181 & 0.019 & -0.200 \\
\hline
\end{tabular}

The transfer of a large number of electrons during adsorption causes the redistribution of system charges. The density of states (DOS) near the Fermi level appears to be impure, for example there is a peak in $-0.5 \mathrm{eV}$. And the DOS between HOMO and LUMO changes. Figure 3 shows that these impure states are caused by $\mathrm{SO}_{2}$ adsorption. The $p$ orbitals of $\mathrm{S}$ and $\mathrm{O}$ atoms have a large overlap with the $d$ orbitals of $\mathrm{Pt}$ atom, and it demonstrates that $\mathrm{SO}_{2}$ can strongly hybridize with $\mathrm{Pt}$ [23]. This has significant effect on the frontier orbital of the adsorption system, which changes the HOMO and LUMO orbital formation, causing the change in the DOS. Figure 4a shows that the $p$ orbitals of $\mathrm{C} 1$ and $\mathrm{C} 3$ form $\sigma$ bond with S. In Figure 4b, the $d$ orbitals of Pt and the $p$ orbitals of S are hybridized. 
The frontier orbital energy gap $E_{\mathrm{g}}$ of the system is $0.285 \mathrm{eV}$ after adsorbing $\mathrm{SO}_{2}$, which is reduced by $0.047 \mathrm{eV}$ compared with that in the non-adsorbed $\mathrm{SO}_{2}$. This is beneficial for the transfer of electrons between HOMO and LUMO, thereby enhancing conductivity.

Figure 3. The density of states of Pt-SWCNTs before and after $\mathrm{SO}_{2}$ adsorption. (a) The DOS of Pt-SWCNTs; (b) The LDOS of $\mathrm{SO}_{2}$-Pt-SWCNTs and $\mathrm{SO}_{2}$ in $\mathrm{SO}_{2}$-Pt-SWCNTs; (c) The PDOS of Pt in Pt-SWCNTs; (d) The PDOS of Pt, O and $\mathrm{S}$ in $\mathrm{SO}_{2}$-Pt-SWCNTs. (Fermi level is $0 \mathrm{eV}$ ).
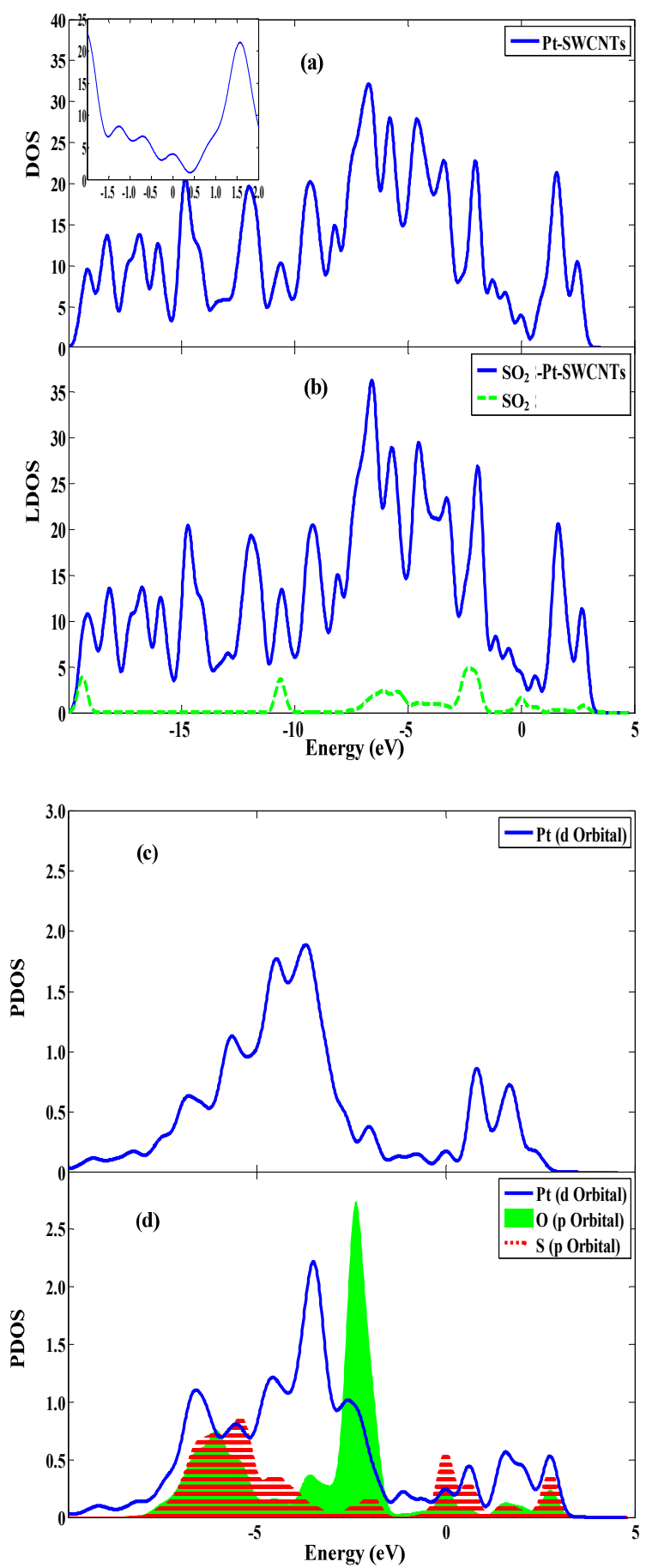
Figure 4. Frontier orbital energy level of $\mathrm{SO}_{2}$-Pt-SWCNTs. (a) $\mathrm{HOMO}(-5.208 \mathrm{eV})$; (b) LUMO $(-4.923 \mathrm{eV})$.
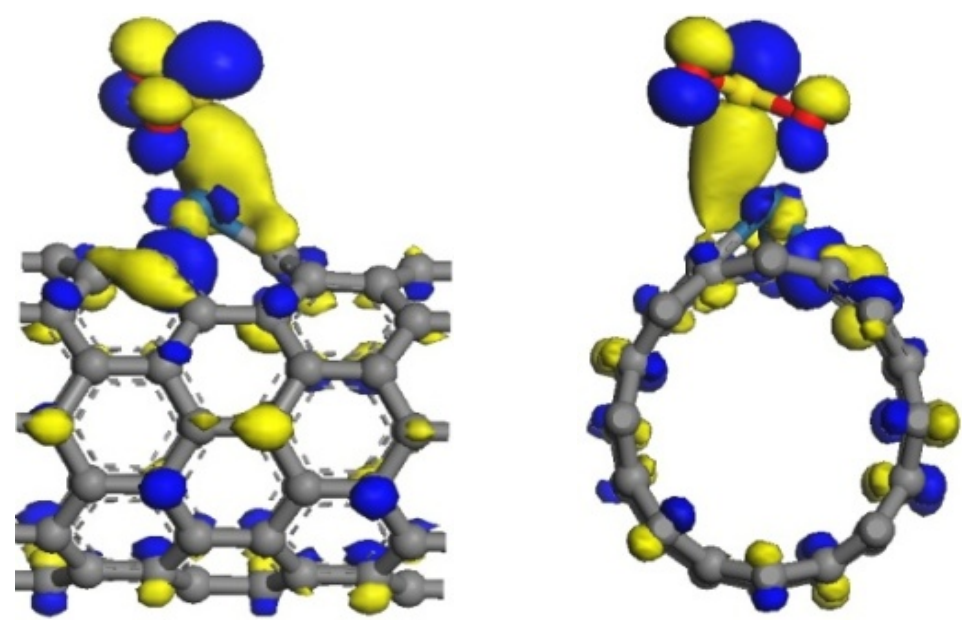

(a)
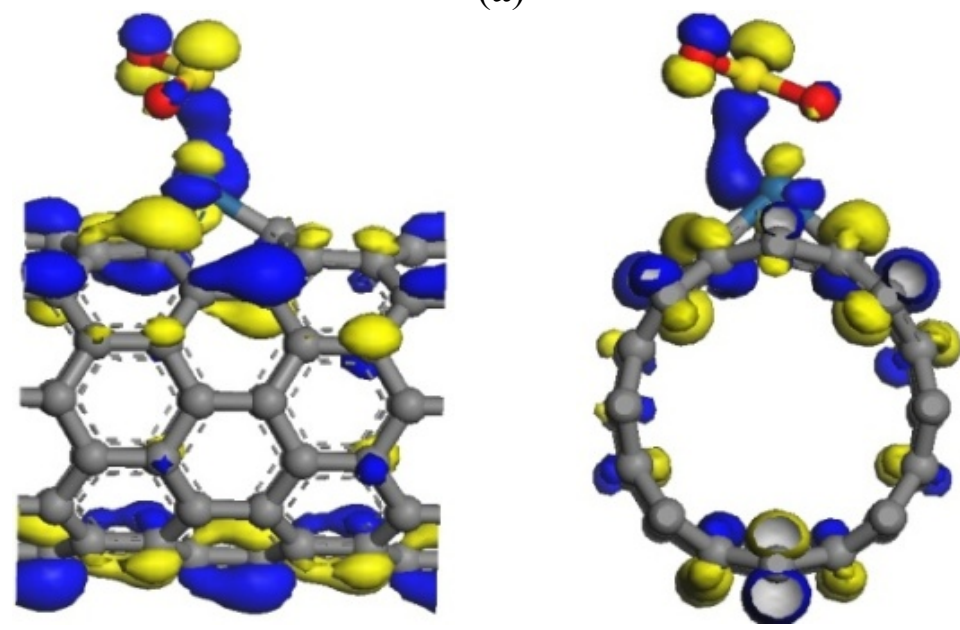

(b)

$\mathrm{SO}_{2}$ adsorption on the surface of Pt-SWCNTs has large adsorption energy and can form a stable structure. The $p$-type Pt-SWCNTs [11] donate electrons and increase the number of hole carriers, reducing the frontier orbital energy, diminishing energy gap $E_{\mathrm{g}}$, and enhancing conductivity. Pt-SWCNTs are highly responsive to $\mathrm{SO}_{2}$, the doped $\mathrm{Pt}$ effectively improved the adsorption sensitivity of SWCNTs to $\mathrm{SO}_{2}$.

\section{2. $\mathrm{H}_{2} \mathrm{~S}$}

$\mathrm{H}_{2} \mathrm{~S}$ which is the simplest hydride of sulfur, is a colorless toxic gas that smells like rotten eggs and is strongly corrosive. It is also harmful to human health. The $\mathrm{S}$ in $\mathrm{H}_{2} \mathrm{~S}$ is at the lowest valence state, so it is strongly reducible.

The adsorption reaction of Pt-SWCNTs and $\mathrm{H}_{2} \mathrm{~S}$ is also exothermic, with $E_{b}$ of $-0.977 \mathrm{eV}$, more than intrinsic SWCNTs $(-0.591 \mathrm{eV}$ in Table 2). The frontier orbital energy differences are $E_{H-L}=4.438 \mathrm{eV}$ and $E_{L-H}=1.519 \mathrm{eV}$, therefore $\mathrm{H}_{2} \mathrm{~S}$ provides electrons to Pt-SWCNTs in this reaction. Mulliken charge analysis (Table 3) shows that $\mathrm{H}_{2} \mathrm{~S}$ donates 0.285 electrons almost 22 times more than intrinsic SWCNTs. Pt and SWCNTs have 0.019 and 0.266 electrons, respectively, after 
$\mathrm{H}_{2} \mathrm{~S}$ is adsorbed on Pt-SWCNTs (Figure 5). A large number of electron transformations convert Pt-SWCNTs from $p$-type to $n$-type. $E_{g}$ of the adsorption system is $0.283 \mathrm{eV}$, which is reduced by $0.049 \mathrm{eV}$ compared that with non-adsorbed $\mathrm{H}_{2} \mathrm{~S}$, thus enhancing conductivity. $\mathrm{H}_{2} \mathrm{~S}-\mathrm{Pt}-\mathrm{SWCNT}$ frontier orbitals concentrate on Pt-SWCNTs, and $\mathrm{H}_{2} \mathrm{~S}$ is not involved in the composition of HOMO and LUMO orbitals. Figure 6 shows that the DOS of $\mathrm{H}_{2} \mathrm{~S}$ is not distributed between the HOMO and LUMO, and the DOS near the Fermi level is basically the same as that of Pt-SWCNTs, which is consistent with the results of frontier orbitals (Figure 7). The $p$ orbitals of $\mathrm{S}$ have a large overlap with the $d$ orbitals of $\mathrm{Pt}$, and the strong interaction of them enhances the adsorption between $\mathrm{H}_{2} \mathrm{~S}$ and the nanotube surface.

Figure 5. Structural model of $\mathrm{H}_{2} \mathrm{~S}-\mathrm{Pt}-\mathrm{SWCNT}$ adsorptive system. (a) Front view; (b) side view.

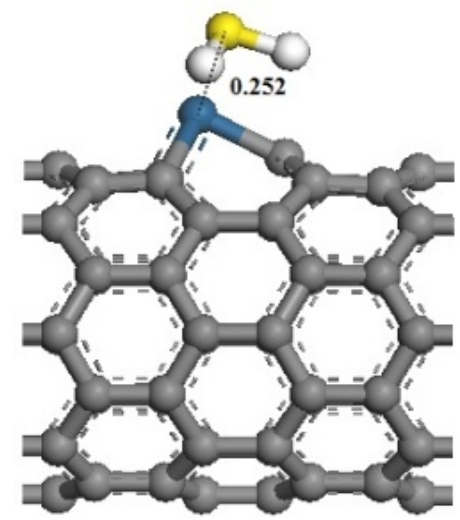

(a)

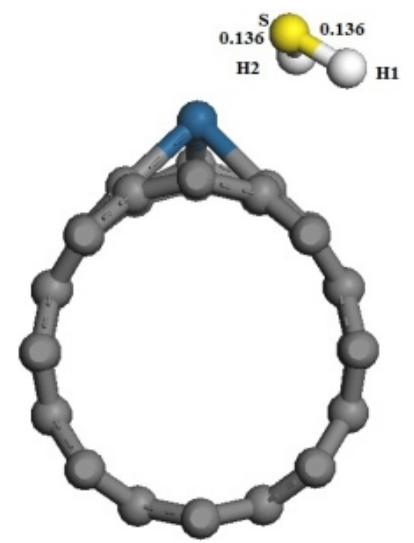

(b)

From the comparison results of adsorption energy and transfer charge, it can be seen that doped $\mathrm{Pt}$ obviously improves the adsorption ability of the instrinsic SWCNTs to $\mathrm{H}_{2} \mathrm{~S} . \mathrm{H}_{2} \mathrm{~S}$ adsorbs on the surface of Pt-SWCNTs and donates substantial electrons to Pt-SWCNTs, which converts CNTs from $p$-type to $n$-type. The frontier orbital energy is increased, while the conductivity is enhanced because of the decrease in the frontier orbital energy gap.

Figure 6. The density of states of $\mathrm{H}_{2} \mathrm{~S}-\mathrm{Pt}-\mathrm{SWCNTs}$. (a) The DOS of $\mathrm{H}_{2} \mathrm{~S}-\mathrm{Pt}-\mathrm{SWCNTs}$ and the LDOS of $\mathrm{H}_{2} \mathrm{~S}$ in $\mathrm{H}_{2} \mathrm{~S}$-Pt-SWCNTs. (b) The PDOS of Pt and $\mathrm{S}$ in $\mathrm{H}_{2} \mathrm{~S}$-Pt-SWCNTs.

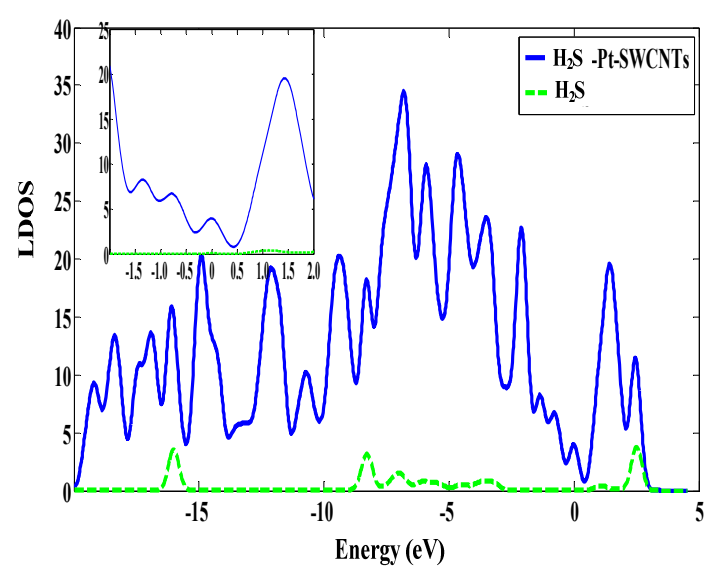

(a)

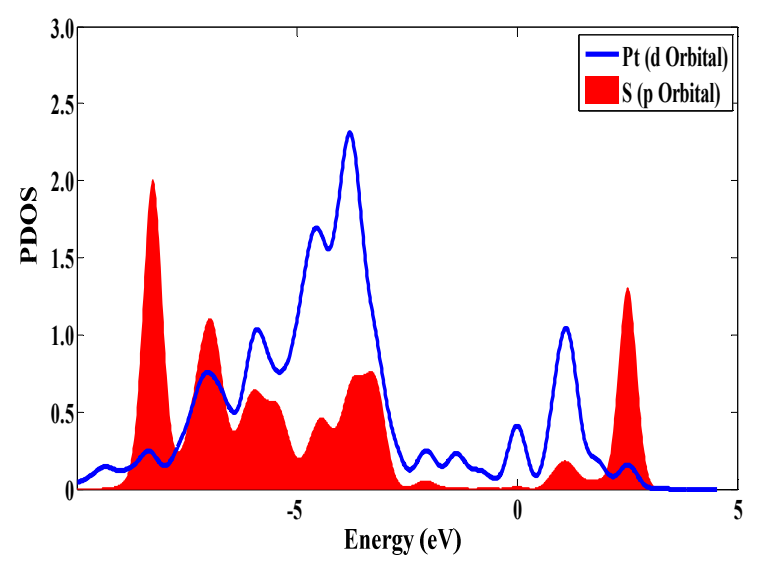

(b) 
Figure 7. Frontier orbital energy level of $\mathrm{H}_{2} \mathrm{~S}-\mathrm{Pt}-\mathrm{SWCNTs}$. (a) $\mathrm{HOMO}(-4.425 \mathrm{eV})$; (b) LUMO $(-4.142 \mathrm{eV})$.
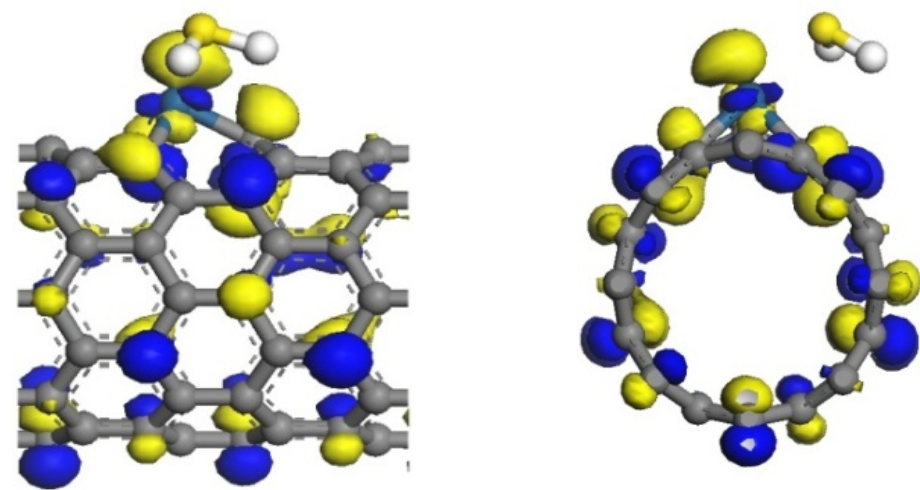

(a)
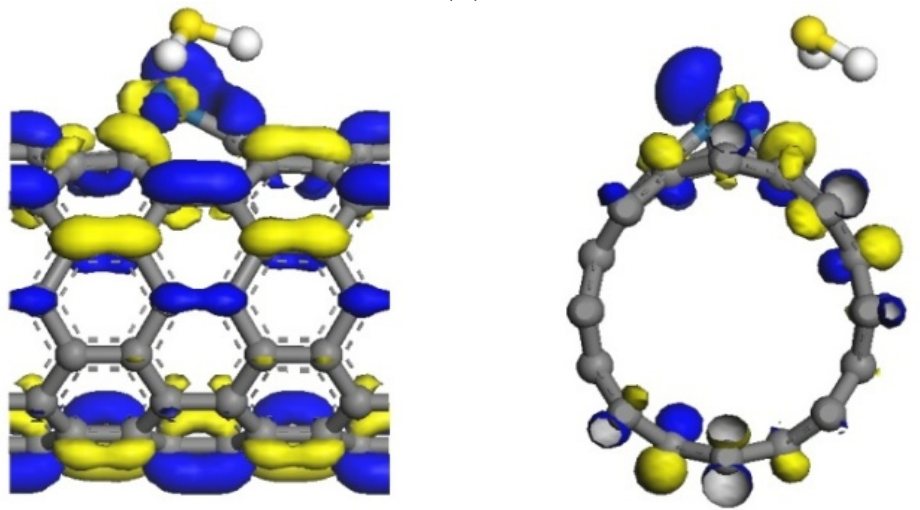

(b)

\section{3. $\mathrm{CO}$}

$\mathrm{CO}$ is a colorless, non-irritating gas. However when it enters the human body, $\mathrm{CO}$ combines with blood hemoglobin, which prevents the union of hemoglobin and oxygen, leading to body tissue hypoxia and even suffocation. The $\mathrm{C}$ atom in $\mathrm{CO}$ has +2 valence electrons and that can be further oxidized to +4 . Accordingly, $\mathrm{CO}$ is a reducing gas that provides electrons in reactions.

The optimized adsorption structure of $\mathrm{CO}$ adsorbed on Pt-SWCNTs is shown in Figure 8, which is consistent with reference [24], $\mathrm{C}$ atoms point to $\mathrm{Pt}$, whereas $\mathrm{O}$ atoms point away from the CNT surface. The adsorption reaction is exothermic. High adsorption energy results in a tight bond between gases and CNTs, as well as an interaction distance of $0.198 \mathrm{~nm}$.

The same as the previous two gases, the adsorption energy and the transfer charge between Pt-SWCNTs and CO are increased obviously, enhancing the adsorption. Figure 9 shows that the $p$ orbitals of $\mathrm{C}$ in $\mathrm{CO}$ have an overlap with the $d$ orbitals of Pt, especially near the Fermi level. The DOS near the Fermi level is changed, and the peak at $-7 \mathrm{eV}$ is split, which is related to $\mathrm{CO}$ adsorption. The contributions of CO to HOMO and LUMO are mainly on the $p$ orbitals of C and $\mathrm{O}$ atoms (Figure 10), which change the system configuration of frontier orbitals, changing the DOS of the system. During adsorption, CO provides 0.181 electrons (Table 3), $p$-type CNTs obtain electrons, and the number of hole carriers decreases. Frontier orbital energy and energy gap $E_{\mathrm{g}}$ increase, decreasing conductivity. 
Figure 8. Structural model of CO-Pt-SWCNT adsorptive system. (a) Front view; (b) side view.

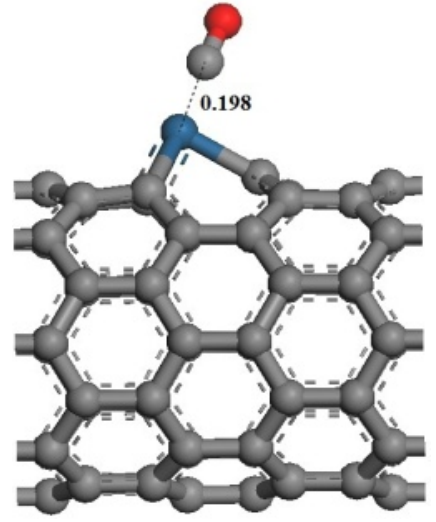

(a)

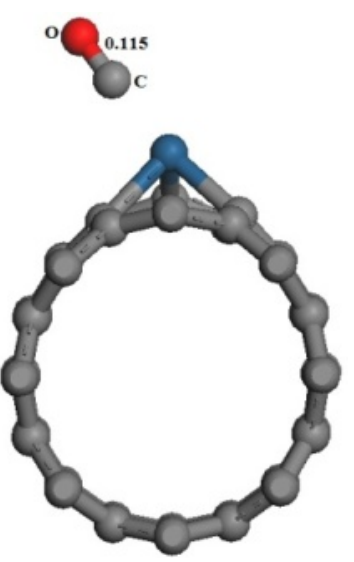

(b)

Figure 9. The density of states of CO-Pt-SWCNTs. (a) The DOS of CO-Pt-SWCNTs and the LDOS of CO in CO-Pt-SWCNTs. (b) The PDOS of Pt and C in CO-Pt-SWCNTs.

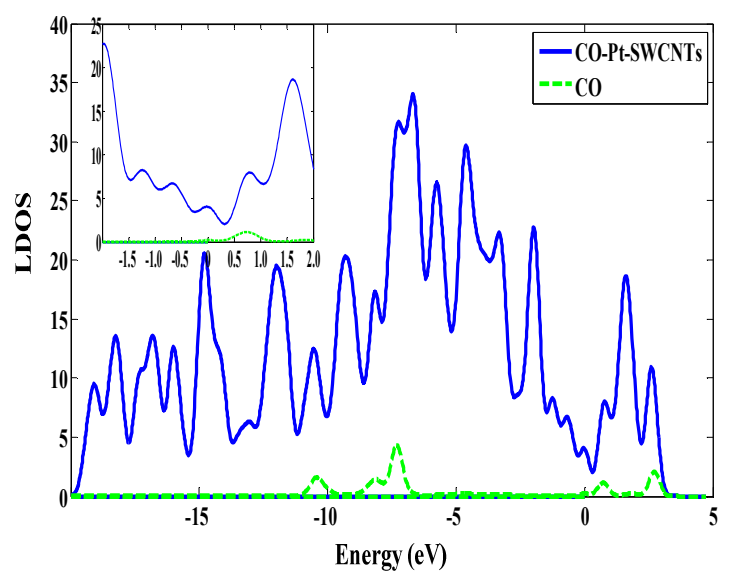

(a)

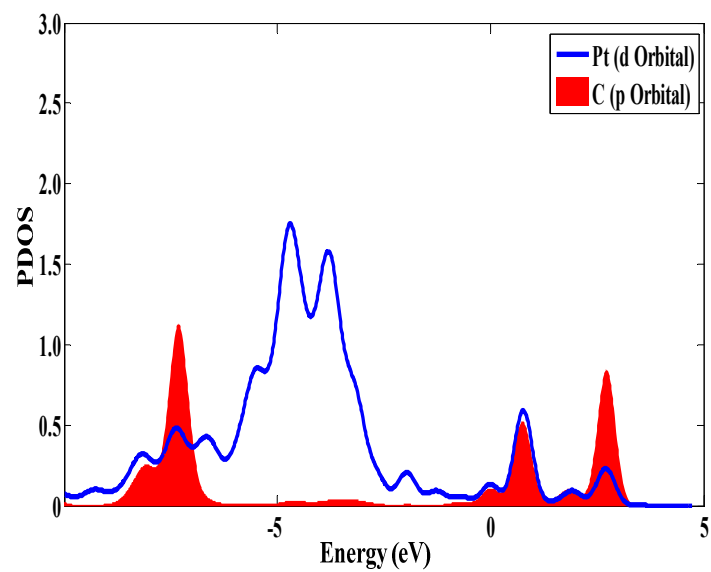

(b)

Figure 10. Frontier orbital energy level of CO-Pt-SWCNTs. (a) HOMO $(-4.797 \mathrm{eV})$; (b) LUMO $(-4.455 \mathrm{eV})$.
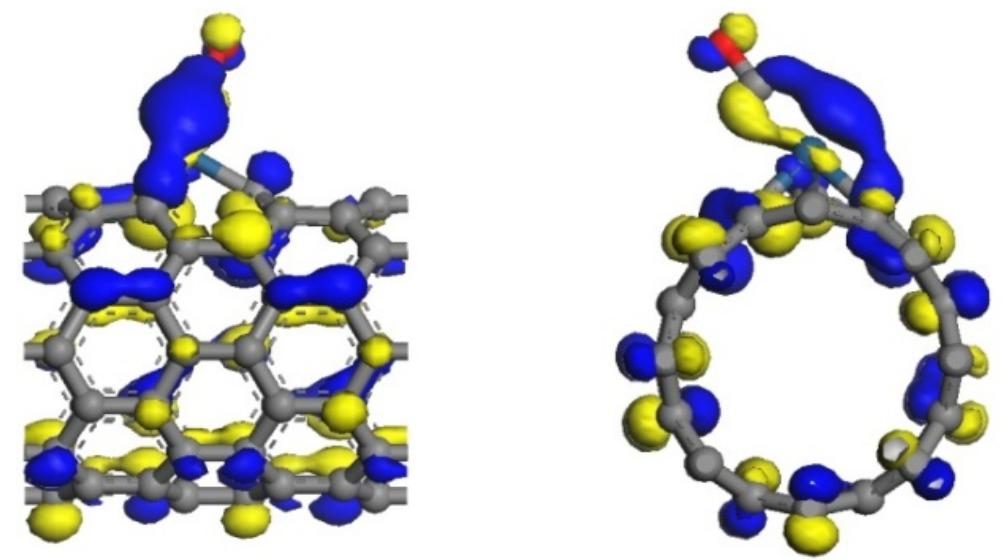

(a) 
Figure 10. Cont.
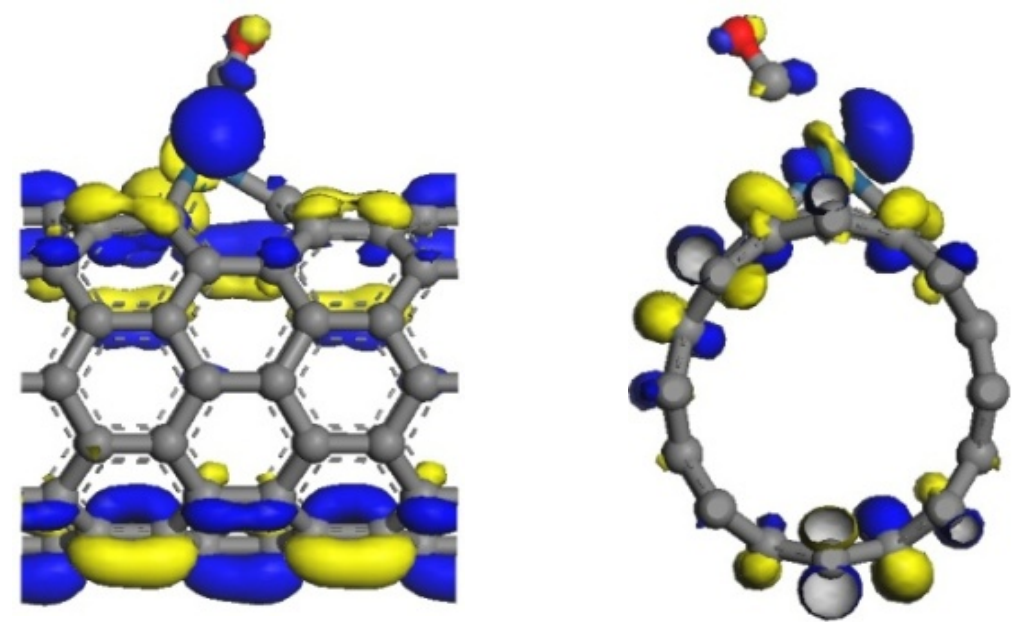

(b)

\section{Discussion}

Pt is a heavy metal with an atomic number of 78 . Its outer core $5 d$ orbitals have nine electrons and an unpaired $d$ electron, so it easily absorbs electrons to reach a steady state. Pt doped into SWCNTs obtains electrons from C. In Pt-SWCNTs, Pt has 0.147 electrons, C1, C2, and C3 adjacent to Pt have 0.028, 0.029, and 0.097 electrons, respectively, which form an electron accumulation zone around the Pt atom. Given that Pt easily obtains electrons, when $\mathrm{SO}_{2}$ reacts with Pt-SWCNTs, SWCNTs donate most of the electrons, and a small charge change in $\operatorname{Pt}\left(\Delta Q_{P t}\right)$ occurs. On the contrary, when the target gases are $\mathrm{H}_{2} \mathrm{~S}$ and $\mathrm{CO}$, Pt exhibits a large charge change.

\section{Conclusions}

In this study, the adsorptions of three gases on the surface of Pt-SWCNTs were calculated based on DFT. The gas-sensing properties of Pt-SWCNTs were assessed according to the changes in adsorption energy, geometric structure, and electronic structure during adsorption. The main conclusions are as follows:

1. The doped Pt effectively improves the adsorption sensitivity of intrinsic SWCNTs to the three kind of gases.

2. The adsorption energy of the reaction between Pt-SWCNTs and $\mathrm{SO}_{2}$ is large, and numerous electrons are transferred from CNTs to the target gases. The frontier orbital energies $\left(E_{H O M O}\right.$ and $\left.E_{L U M O}\right)$ and $E_{\mathrm{g}}$ are decreased, and the electrical conductivity of Pt-SWCNTs is enhanced. Pt-SWCNTs have high sensitivity to $\mathrm{SO}_{2}$.

3. $\mathrm{H}_{2} \mathrm{~S}$ is reduced when reacted with Pt-SWCNTs. $\mathrm{H}_{2} \mathrm{~S}$ provides a large number of electrons, converting CNTs from $p$-type to $n$-type. The frontier orbital energies are increased, whereas $E_{\mathrm{g}}$ is decreased, thereby enhancing conductivity.

4. When CO is adsorbed on Pt-SWCNTs, CO provides electrons to $p$-type CNTs, decreasing the number of hole carriers. The frontier orbital energies and $E_{\mathrm{g}}$ are increased, decreasing conductivity. 
Results of the theoretical calculation show that Pt-SWCNTs can respond to the three gases. The electrical characteristics of Pt-SWCNTs show different degrees of changes after adsorption of the test gases. As $\mathrm{SO}_{2}$ is adsorbed on Pt-SWCNTs, the CNTs lose electrons, the number of hole carriers is increased, and conductivity is enhanced. $\mathrm{As}_{2} \mathrm{~S}$ is adsorbed on the surface of CNTs, Pt-SWCNTs receive a large number of electrons and transform from $p$-type into $n$-type. The conductivity of Pt-SWCNTs is also enhanced. Comparing their adsorption energies and charge transformations, the sensitivity to $\mathrm{SO}_{2}$ is higher than the sensitivity of Pt-SWCNTs to $\mathrm{H}_{2} \mathrm{~S}$. Moreover, $\mathrm{CO}$ is an electron-donor gas, which reduces hole carriers and weakens conductivity. Therefore, Pt-SWCNTs can be used to fabricate gas sensors in detecting $\mathrm{SO}_{2}, \mathrm{H}_{2} \mathrm{~S}$, and $\mathrm{CO}$ gases.

\section{Acknowledgments}

We gratefully acknowledge the financial support from Project No. 0213005202042, which is supported by the Fundamental Research Funds for the Central Universities.

\section{Conflict of Interest}

The authors declare no conflict of interest.

\section{References}

1. Zhang, X.-X.; Liu, W.-T.; Tang, J.; Xiao, P. Study on PD detection in $\mathrm{SF}_{6}$ using multi-wall carbon nanotube films sensor. IEEE Trans. Dielectr. Electr. Insul. 2010, 17, 838-844.

2. Kong, J.; Franklin, N.R.; Zhou, C.; Chapline, M.G.; Peng, S.; Kyeongjae, C.; Hongjie, D. Nanotube molecular wires as chemical sensors. Science 2000, 287, 622-625.

3. Zhang, X.-X.; Bing, Y.; Wang, X.-J.; Luo, C.-C. Effect of plasma treatment on multi-walled carbon nanotubes for the detection of $\mathrm{H}_{2} \mathrm{~S}$ and $\mathrm{SO}_{2}$. Sensors 2012, 12, 9375-9385.

4. Zhang, X.-X.; Bing, Y.; Dai Z.-Q.; Luo C.-C. The gas response of hydroxyl modified SWCNTs and carboxyl modified SWCNTs to $\mathrm{H}_{2} \mathrm{~S}$ and $\mathrm{SO}_{2}$. Przeglad Elektrotechniczny 2012, 88, 311-314.

5. Zhao, Z.; Buldum, A.; Han, J.; Lu, J.P. Gas molecule adsorption in carbon nanotubes and nanotube bundles. Nanotechnology 2002, 13, 195-200.

6. Collins, P.G.; Bradley, K.; Ishigami, M.; Zettl, A. Extreme oxygen sensitivity of electronic properties of carbon nanotubes. Science 2000, 287, 1801-1804.

7. Goldoni, A.; Larciprete, R.; Petaccia, L.; Lizzit, S. Single-wall carbon nanotube interaction with gases: Sample contaminants and environmental monitoring. J. Am. Chem. Soc. 2003, 124, 11329-11333.

8. Kemp, K.C.; Seema, H.; Saleh, M.; Le, N.H.; Mahesh, K.; Chandra, V.; Kim, K.S. Environmental applications using graphene composities: Water remediation and gas adsorption. Nanoscale 2013, 5, 3149-3171.

9. Peng, S.; Cho, K. Ab initio study of doped carbon nanotube sensors. Nano Lett. 2003, 3, 513-517. 
10. Kim, S.J.; Park, Y.J.; Ra, E.J.; Kim, K.K.; An, K.H.; Lee, Y.H.; Choi, J.Y.; Park, C.H.; Doo, S.K.; Park, M.H.; Yang, C.W. Defect-induced loading of Pt nanoparticles on carbon nanotubes. Appl. Phys. Lett. 2007, 90, 023114/1-023114/3.

11. Pannopard, P.; Khongpracha, P.; Probst, M.; Limtrakul, J. Gas sensing properties of platinum derivatives of single-walled carbon nanotubes: A DFT analysis. J. Mol. Graph. Model. 2009, 28, 62-69.

12. Penza, M.; Cassano, G.; Rossi, R.; Alvisi, M.; Rizzo, A.; Signore, M.A.; Dikonimos, Th.; Serra, E.; Giorgi, R..Enhancement of sensitivity in gas chemiresistors based on carbon nanotube surface functionalized with noble metal (Au, Pt) nanoclusters. Appl. Phys. Lett. 2007, 90, 173123/1-173123/3.

13. Wang, Y.; Balbuena P.B. Roles of proton and electric field in the electroreduction of $\mathrm{O}_{2}$ on $\mathrm{Pt}$ (111) surfaces: Results of an ab-initio molecular dynamics study. J. Phys. Chem. B 2004, 108, 4376-4384.

14. Grabow, L.C.; Gokhale, A.A.; Evans, S.T.; Dumesic, J.A.; Mavrikakis, M. Mechanism of the water gas shift reaction on Pt: First principles, experiments, and microkinetic modeling. J. Phys. Chem. C 2008, 112, 4608-4617.

15. Zhou, C.; Wu, J.; Nie, A.; Forrey, R.C.; Tachibana, A.; Cheng, H. On the sequential hydrogen dissociative chemisorption on small platinum clusters: A density functional theory study. J. Phys. Chem. C 2007, 111, 12773-12778.

16. Orita, H.; Inada, Y. DFT investigation of CO adsorption on Pt (211) and Pt (311) surfaces from low to high coverage. J. Phys. Chem. B 2005, 109, 22469-22475.

17. Tiwari, J.N.; Nath, K.; Kumar, S; Tiwari, R.N.; Kemp, K.C.; Le, N.H.; Youn, D.H.; Lee, J.S.; Kim, S.K.. Stable platinum nanoclusters on genomic DNA-graphene oxide with a high oxygen reduction reaction activity. Nat. Commun. 2013, 4, 1-7.

18. Perdew, J.P.; Wang, Y. Accurate and simple analytic representation of the electron-gas correlation energy. Phys. Rev. B 1992, 45 13244-13249.

19. Delley, B. Hardness conserving semilocal pseudopotentials. Phys. Rev. B 2002, 66, 155125/1-155125/9.

20. Monkhorst, H.J.; Pack, J.D. Special points for Brillouin-zone integrations. Phys. Rev. B 1976, 13, 5188-5192.

21. Srivastava, D.; Menon, M.; Cho, K. Nanoplasticity of single-wall carbon nanotubes under uniaxial compression. Phys. Rev. Lett. 1999, 83, 2973-2976.

22. Park, Y.; Lahaye, R.J.W.E.; Lee, Y.H. Adsorption of Pt on defective carbon nanotube walls: A DFT approach. Comput. Phys. Commun. 2007, 177, 46-46.

23. Ganji, M.D.; Sharifi, N.; Ardjmand, M.; Ahangari, M.G. Pt-decorated grapheme as superior media for $\mathrm{H}_{2} \mathrm{~S}$ adsorption: A first-principles study. Appl. Surf. Sci. 2012, 261, 697-704.

24. Yeung, C.S.; Liu, L.V.; Wang, Y.A. Adsorption of small gas molecules onto Pt-doped single-walled carbon nanotubes. J. Phys. Chem. C 2008, 112, 7401-7411.

(C) 2013 by the authors; licensee MDPI, Basel, Switzerland. This article is an open access article distributed under the terms and conditions of the Creative Commons Attribution license (http://creativecommons.org/licenses/by/3.0/). 